Portland State University

PDXScholar

Electrical and Computer Engineering Faculty

Publications and Presentations

$5-1-1975$

\title{
Gaussian Modes in High Loss Laser Resonators
}

Lee W. Casperson

Portland State University

Susan D. Lunnam

University of California, Los Angeles

Follow this and additional works at: https://pdxscholar.library.pdx.edu/ece_fac

Part of the Electrical and Computer Engineering Commons

Let us know how access to this document benefits you.

\section{Citation Details}

Lee W. Casperson and Susan D. Lunnam, "Gaussian Modes in High Loss Laser Resonators," Appl. Opt. 14, 1193-1199 (1975).

This Article is brought to you for free and open access. It has been accepted for inclusion in Electrical and Computer Engineering Faculty Publications and Presentations by an authorized administrator of PDXScholar. Please contact us if we can make this document more accessible: pdxscholar@pdx.edu. 


\title{
Gaussian Modes in High Loss Laser Resonators
}

\author{
Lee W. Casperson and Susan D. Lunnam
}

\begin{abstract}
Matrix techniques are applied to the mode analysis of laser resonators having spherical mirrors and Gaussian profiles of the mirror reflectivity. These same analytical methods provide a useful approximation to the mode and loss characteristics of conventional resonators having an abrupt discontinuity of the reflectivity. Mode selection in apertured waveguides and resonators is also discussed.
\end{abstract}

\section{Introduction}

There has recently been substantial interest in the development of laser resonators having large diffraction losses at the laser mirrors. Such resonators can be used to obtain oscillation in the fundamental TEM $_{00}$ transverse mode because of the much greater losses of the higher order modes. This kind of mode discrimination is especially important in lasers with high gain amplifiers such as $\mathrm{CO}_{2}$ TEA devices. Also, the radiation that is lost from the cavity is available as the laser output, and diffraction coupling can reduce the energy transmission requirements of the mirror coatings and substrates.

The simplest high loss resonators consist of highly reflecting mirrors in which the reflectivity drops abruptly to zero outside of a well-defined radius. The modes and losses of such resonators are usually found using iterative solutions of the diffraction integrals based on the Fox and $\mathrm{Li}^{1}$ or Prony ${ }^{2}$ methods. More recently resonators have been developed having graded reflection profiles and shaped apertures. ${ }^{3}$ The advantage of tapering the reflectivity is that more desirable output beam modes can be obtained. A useful specific type of profile is one in which the reflectivity has a Gaussian radial variation. It has been found that the modes of such resonators can be described analytically in terms of well-known Hermite-Gaussian ${ }^{4}$ or Laguerre-Gaussian ${ }^{5}$ functions. In this work it is shown that the Gaussian modes of these resonators may be obtained in a straightforward way using matrix techniques. The procedure applies to unsymmetric resonators having unequal mirror curvatures and reflectivity profiles and containing arbitrary sequences of lenses and complex lenslike media. The matrix method is simpler than

The authors are with the School of Engineering \& Applied Science, University of California, Los Angeles, California 90024.

Received 16 September 1974. manipulation of the diffraction integrals, and the inclusion of a lenslike medium is required for a rigorous analysis of many high loss lasers. Stability conditions are given for determining the sensitivity of the resonator to perturbations.

Also, the Gaussian mode formalism is investigated as a means of approximating the modes and diffraction losses of conventional resonators with abrupt reflectivity discontinuities. The Gaussian modes are similar to the computer derived modes, and the analytic loss expressions are accurate to within a few percent. Thus the Gaussian approximation is a powerful practical technique for estimating resonator behavior. In addition computer time should be saved by using these modes as a first estimate for more precise numerical mode iterations. Last, the matrix methods are used to investigate the stability and mode selection characteristics of lens and aperture waveguides. Under some conditions a sequence of lens elements and apertures is formally equivalent to a complex lenslike medium.

\section{Gaussian Graded Mirrors and Apertures}

A well-known and efficient means for analyzing the propagation of Gaussian light beams involves the use of $2 \times 2 A B C D$ matrices. ${ }^{6}$ These beam matrices may be used to characterize a variety of simple optical elements including lenses, spherical mirrors, and quadratic lenslike media. Propagation through a sequence of such elements is governed simply by the product beam matrix according to the transformation

$$
\frac{1}{q(2)}=\frac{C+D / q(1)}{A+B / q(1)}
$$

Here $q(1)$ and $q(2)$ are, respectively, the input and output values of the complex beam radius. This radius is related to the radius of curvature of the phase fronts $R$ and the spot size $w$ by

$$
\frac{1}{q}=\frac{1}{R}-\frac{i \lambda}{\pi w^{2}}
$$


where $\lambda$ is the wavelength in the medium. Higher order Hermite-Gaussian and Laguerre-Gaussian modes are governed by the same parameters. If the properties of the system are different in the $x$ direction from in the $y$ direction it is necessary to treat separately the parameters $q_{x}$ and $q_{y}$.

The modes of resonators containing only simple optical elements of the types mentioned previously may be found from the self-consistency requirement that $q$ repeat itself after one round trip through the resonator. If the round trip matrix elements are $A$, $B, C, D$, the steady-state beam parameter $q_{s}$ at the chosen reference plane must be a solution of

$$
\frac{1}{q_{s}}=\frac{C+D / q_{s}}{A+B / q_{s}}
$$

This is a quadratic equation having the two roots

$$
\frac{1}{q_{s}}=\frac{D-A}{2 B} \pm \frac{1}{B}\left[\left(\frac{A+D}{2}\right)^{2}-1\right]^{1 / 2},
$$

where we have used the determinantal relation $A D-$ $B C=1$ which applies to all optical resonators.

These matrix techniques may sometimes be applied even though the mirror reflectivity is not constant over the mirror surface. We consider first the case of an aperture having a Gaussian radial transmission profile $t(r)$ governed by

$$
t(r)=t_{0} \exp \left(-r^{2} / w_{m}{ }^{2}\right),
$$

where $t_{0}$ is the amplitude transmission at the center of the aperture, and $w_{m}$ is the spot size. If the amplitude distribution of the incident beam is the Gaussian function

$$
E(r)=E_{0} \exp \left(-r^{2} / w_{i}^{2}\right),
$$

it follows from Eqs. (5) and (6) that the spot size $w_{t}$ of the transmitted beam is related to $w_{i}$ by

$$
\frac{1}{w_{t}^{2}}=\frac{1}{w_{i}^{2}}+\frac{1}{w_{m}^{2}} \text {. }
$$

Therefore, the transmission of a Gaussian beam through the aperture is governed by Eq. (1) provided that the aperture matrix is

$$
\left(\begin{array}{cc}
1 & 0 \\
-\frac{i \lambda}{\pi u_{m}^{2}} & 1
\end{array}\right) .
$$

A related matrix has been employed previously in the analysis of stabilizing apertures for optical waveguides. ${ }^{7}$ Some basic beam matrices are shown for reference in Fig. 1. The matrix for an optical system is the ordered product of the matrices corresponding to the individual optical elements. Thus the matrix for a Gaussian graded mirror is the product of matrices (3) and (5).

Off-axis beams can be treated in terms of the same formalism. The propagation of a transverse Cartesian field component of an astigmatic off-axis Gauss- ian beam may be described in terms of the exponential function ${ }^{8}$

$$
\begin{aligned}
& G(x, y, z)=\exp \\
& -i\left[\frac{Q_{x}(z) x^{2}}{2}+\frac{Q_{y}(z) y^{2}}{2}+S_{x}(z) x+S_{y}(z) y+P(z)\right],
\end{aligned}
$$

where it is assumed that the principal axes of the beam are parallel to the $x$ and $y$ axes. It follows from Eq. (9) that the displacement of the beam in the $x$ direction is equal to $d_{x a}=-S_{x i} / Q_{x i}$, and the displacement of the center of the phase fronts is equal to $d_{x p}=-S_{x r} / Q_{x r}$, where the subscripts $i$ and $r$ refer, respectively, to the imaginary and real parts of the functions $S_{x}$ and $Q_{x}=2 \pi / \lambda q_{x}$. The propagation of the parameters $q_{x}$ and $q_{y}$ of an off-axis beam in a system of elementary lenses and lenslike media is governed by Eq. (3), while the propagation of the $S$ parameters is governed by ${ }^{9}$

$$
S_{x}(2)=\frac{S_{x}(1)}{A+B / q_{x}(1)} .
$$

One readily finds that this same transformation law also applies to the matrix elements of Gaussian graded apertures. Thus, the propagation of the phase front curvature, spot size, and beam displacement of an off-axis beam in a system containing the lens elements shown in Fig. 1 is determined immediately from the corresponding product matrix. The transformation of the amplitude displacement through an aperture reduces to

$$
d_{x a}(2)=d_{x a}(1)\left\{1+\left[w_{x}(1) / w_{m}\right]^{2}\right\}^{-1} .
$$

With these basic matrix procedures established, we now consider the problem of determining the modes

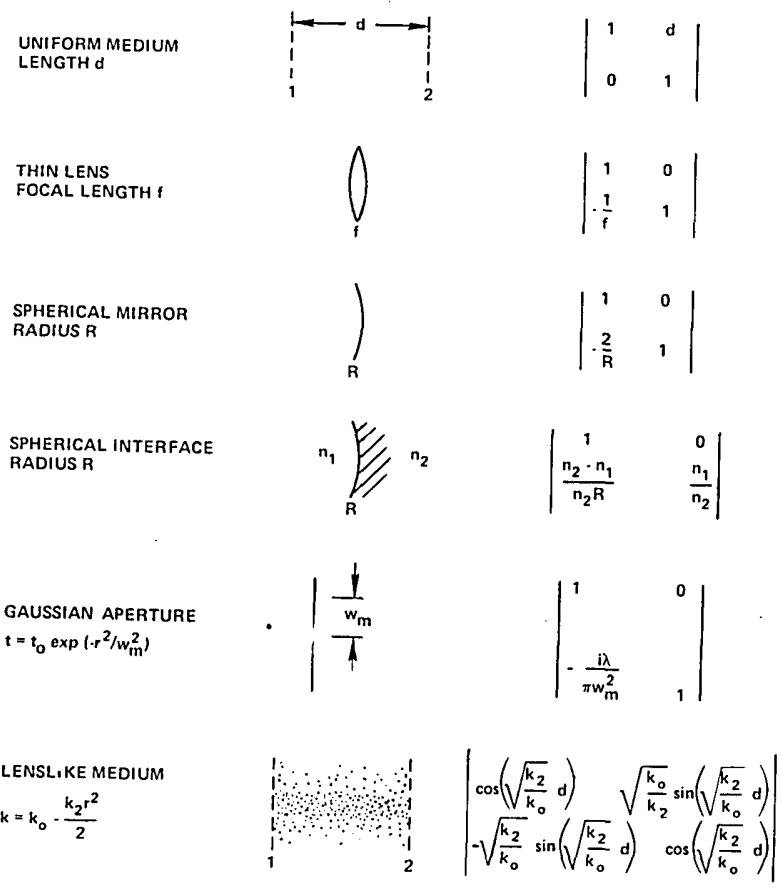

Fig. 1. Matrices for a Gaussian beam incident from the left. 


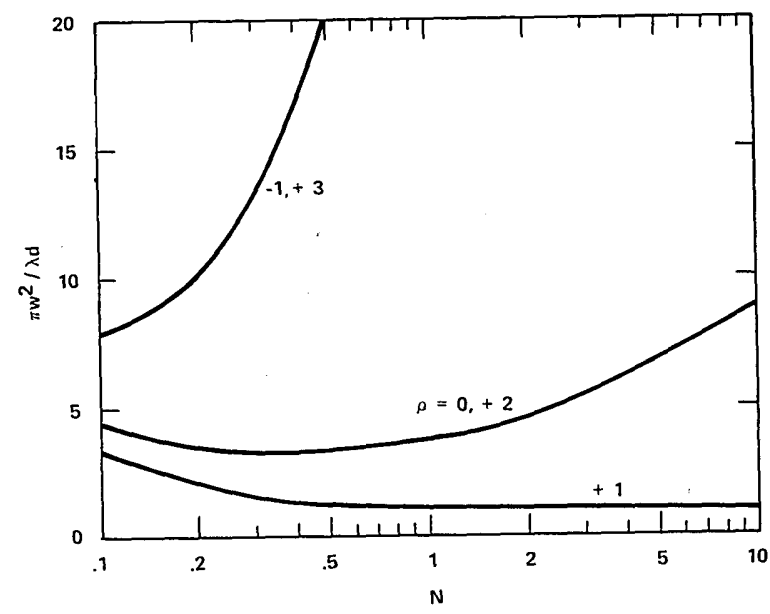

Fig. 2. Normalized spot size $\pi w^{2} / \lambda d$ at the mirror as a function of the Fresnel number $N=w_{m}{ }^{2} / \lambda d$ for various values of $\rho=d / R_{m}$.

of a resonator containing mirrors with Gaussian graded reflectivity. For simplicity and comparison with previous analyses, we restrict attention here to a free space symmetric resonator of length $d$. The governing matrix is the product

$$
\begin{aligned}
& \left(\begin{array}{ll}
A & B \\
C & D
\end{array}\right)=\left(\begin{array}{ll}
1 & d \\
0 & 1
\end{array}\right) \\
& \times\left(\begin{array}{cc}
1 & 0 \\
-\frac{2}{R_{m}}-\frac{i \lambda}{\pi w_{m}^{2}} & 1
\end{array}\right)=\left(\begin{array}{cc}
1-\frac{2 d}{R_{m}}-i \frac{\lambda d}{\pi w_{m}^{2}} & d \\
-\frac{2}{R_{m}}-i \frac{\lambda}{\pi w_{m}^{2}} & 1
\end{array}\right) .
\end{aligned}
$$

From Eq. (4) the complex radius of the beam incident on the output mirror is

$$
\frac{d}{q}=\frac{d}{R_{m}}+\frac{i \lambda d}{2 \pi w_{m}^{2}} \pm\left[\left(1-\frac{d}{R_{m}}-\frac{i \lambda d}{2 \pi w_{m}^{2}}\right)^{2}-1\right]^{1 / 2} \text {. }
$$

Therefore, the phase front curvature and spot size are given by the real and imaginary parts of

$$
\frac{d}{R}-\frac{i \lambda d}{\pi w^{2}}=\rho+\frac{i}{2 \pi N} \pm\left[\left(1-\rho-\frac{i}{2 \pi N}\right)^{2}-1\right]^{1 / 2} \text {, }
$$

where we have defined the Fresnel number of the resonator by $N=w_{m}^{2} / \lambda d$, and $\rho=d / R_{m}$. The arbitrary sign in Eq. (14) must be chosen to yield a confined beam with a real spot size.

Equation (14) can be separated into its real and imaginary parts by using results of the type

$$
\begin{aligned}
(a+i b)^{1 / 2}= \pm 2^{-1 / 2}\left\{\left[\left(a^{2}+b^{2}\right)^{1 / 2}+a\right]^{1 / 2}\right. & \\
& \left.+i\left[\left(a^{2}+b^{2}\right)^{1 / 2}-a\right]^{1 / 2}\right\} .
\end{aligned}
$$

The conjugate relationship applies when the imaginary part is negative. In Figs. 2 and 3 are plots from Eq. (14) of the beam spot size and phase front curvature at the mirror for various values of the parameter $\rho$. The value $\rho=0$ corresponds to a plane parallel resonator, $\rho=1$ is confocal, and $\rho=2$ is concentric. For very small Fresnel numbers the spot size increases due to the large beam diffraction, while for very large Fresnel numbers the spot size approaches the value appropriate to the corresponding resonator with uniform reflectivity mirrors. With the confocal resonator, the phase is uniform across the mirrors for all values of the reflectivity spot size. Simple approximations to Eq. (14) can readily be found for the limits of small or large $N$.

Using the spot size values found previously, it is possible to calculate the fractional power loss experienced by the beam as it reflects from one of the resonator mirrors. If the beam and mirrors have circular symmetry and the reflectivity is unity at the mirror center, the power loss $L_{00}$ of the fundamental TEM 00 Gaussian mode is

$$
\begin{aligned}
& L_{00}=\frac{P_{t}}{P_{i}} \\
& =\frac{I_{0} \int_{0}^{2 \pi} \int_{0}^{\infty} \exp \left(-2 r^{2} / w^{2}\right)\left[1-\exp \left(-2 r^{2} / w_{m}^{2}\right)\right] r d r d \theta}{I_{0} \int_{0}^{2 \pi} \int_{0}^{\infty} \exp \left(-2 r^{2} / w^{2}\right) r d r d \theta},
\end{aligned}
$$

where $I_{0}$ is the maximum intensity and $P_{i}$ and $P_{t}$ are, respectively, the total incident and transmitted power. The integrals are elementary, and the result is

$$
L_{00}=\left(1+w_{m}^{2} / w^{2}\right)^{-1}=\left[1+\pi N\left(\lambda d / \pi w^{2}\right)\right]^{-1} .
$$

A similar treatment applies to the $\mathrm{TEM}_{10}$ mode. The beam parameter is the same as before, and the loss per pass is

$L_{10}$

$=\frac{I_{0} \int_{0}^{2 \pi} \int_{0}^{\infty} \cos ^{2} \theta \exp \left(-2 r^{2} / w^{2}\right)\left[1-\exp \left(-2 r^{2} / w_{m}^{2}\right)\right] r^{3} d r d \theta}{I_{0} \int_{0}^{2 \pi} \int_{0}^{\infty} \cos ^{2} \theta \exp \left(-2 r^{2} / w^{2}\right) r^{3} d r d \theta}$

$=1-\left(1+w^{2} / w_{m}^{2}\right)^{-2}=1-\left[1+(\pi M)^{-1}\left(\pi w^{2} / \lambda d\right)\right]^{-2}$.

Equations (17) and (19) are plotted in Fig. 4. It is clear that the confocal system $\rho=1$ has the lowest losses.

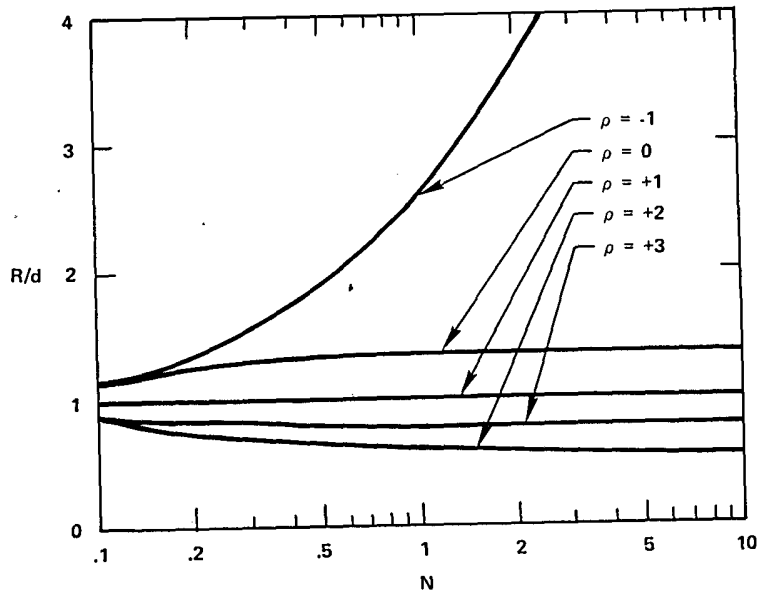

Fig. 3. Normalized phase front curvature $R / d$ at the mirror as a function of the Fresnel number for various values of $\rho=d / R_{m}$. 


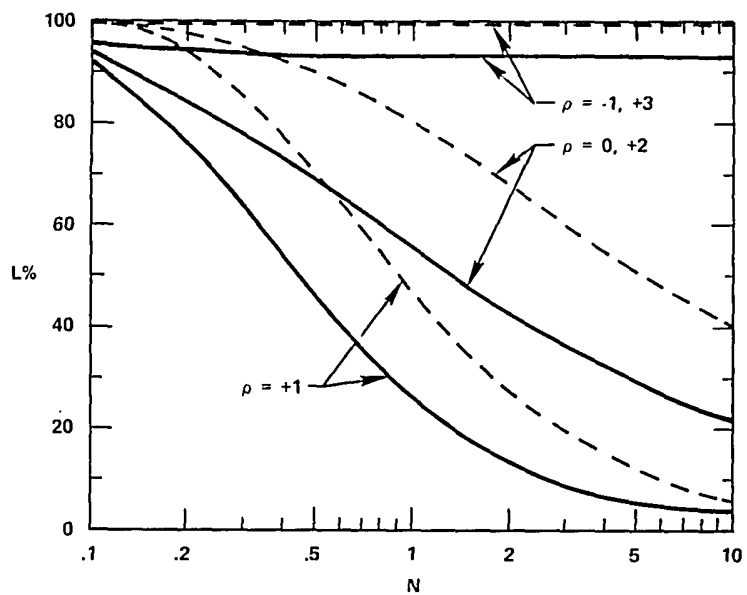

Fig. 4. Power transmission loss at the mirror as a function of the Fresnel number for various values of $\rho=d / R_{m}$. The solid lines indicate loss $L_{00}$ for the fundamental mode, and the dashed lines show the loss $L_{10}$ of the $\mathrm{TEM}_{10}$ mode.

In Fig. 5 is a plot of the mode stability factor ${ }^{9}$

$$
F=|A+B / q| \text {. }
$$

The factor $F^{-2}$ measures the fractional decrease per mirror bounce of any perturbation of the beam parameter $q$. A value $F=2$, for example, implies that any perturbation will be reduced in magnitude to $1 / 4$ of its initial value with each successive reflection. The factor $F^{-1}$ measures the decrease in the beam displacement. The necessary and sufficient condition for stability is always $F \geq 1$. Thus all the resonators discussed here having Gaussian reflectivity profiles support stable confined modes. The stabilizing effect of a mirror with a loss profile is analogous to stabilization due to a gain profile in the laser medium. If the mirror profile were reversed having the reflectivity minimum at the axis, the confined mode solutions would be unstable with respect to perturbations and lasing in the $\mathrm{TEM}_{00}$ mode would not be possible.

\section{Gaussian Approximation for Abrupt Mirror Profiles}

There are several advantages to resonators with graded mirror reflectivities as discussed previously, but conventional abrupt mirror profiles are still easiest to fabricate and may under some conditions have higher damage thresholds. Therefore, abrupt mirror profiles remain of interest, and the purpose of this section is to consider the possibility of applying the previous analytic techniques to the approximate calculation of the properties of high loss conventional laser resonators. In numerical mode studies, it has been observed that the $\mathrm{TEM}_{00}$ and $\mathrm{TEM}_{10}$ fields usually resemble closely their Gaussian counterparts. ${ }^{10}$ Also the detailed mode structure of high loss resonators is often not of practical importance. The quantity of most interest is the loss per pass, and we illustrate the application of the Gaussian methods to the loss calculations for abruptly profiled resonators. Circular mirrors are considered, but the same techniques may be applied to resonators having strip mirrors.

If the mirror diameter is regarded as equivalent to the Gaussian mirror reflectivity spot size, the approximate spot size of the incident beam is given in Fig. 2 for several resonators configurations. Now the losses can be readily calculated. In analogy with Eq. (16), the fractional power transmission of the TEM $\mathrm{T}_{00}$ mode is

$$
L_{00}=\frac{P_{t}}{P_{i}}=\frac{I_{0} \int_{0}^{2 \pi} \int_{w_{m}}^{\infty} \exp \left(-2 r^{2} / w^{2}\right) r d r d \theta}{I_{0} \int_{0}^{2 \pi} \int_{0}^{\infty} \exp \left(-2 r^{2} / w^{2}\right) r d r d \theta} .
$$

The result of the integration is

$$
L_{00}=\exp \left(-2 w_{m}{ }^{2} / w^{2}\right)=\exp \left[-2 \pi N\left(\lambda d / \pi w^{2}\right)\right] .
$$

Similarly, the transmission of the $\mathrm{TEM}_{10}$ mode is

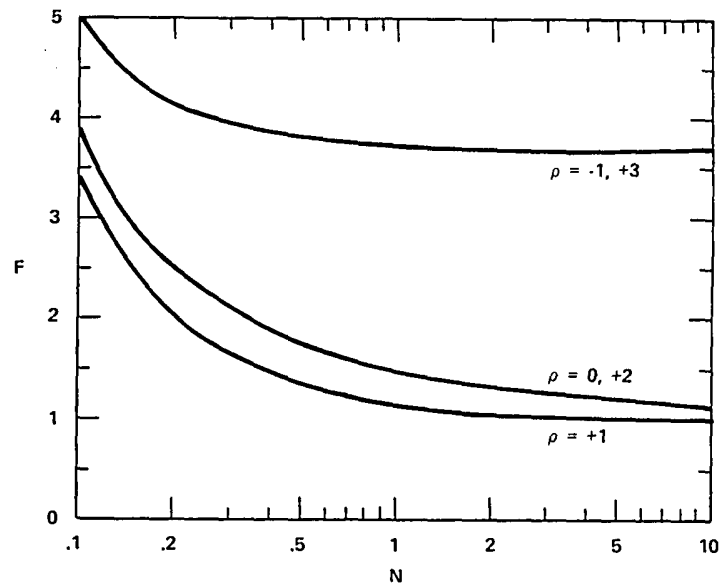

Fig. 5. Stability factor $F=|A+B / q|$ as a function of the Fresnel number for various values of $\rho=d / R_{m}$. Stability is assured by $F \geq 1$.

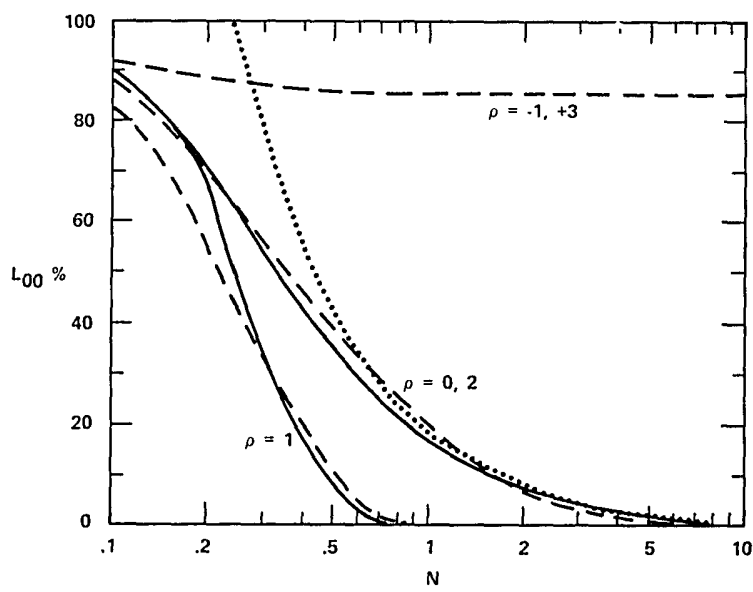

Fig. 6. Power transmission $L_{00}$ of the fundamental TEM 00 mode as a function of the Fresnel number for various values of $\rho=d / R_{m}$. The exact numerical solutions are shown as solid lines, the analytic Gaussian mode approximations are shown as dashed lines, and a previous approximation is shown as a dotted line. 
If this matrix is raised to the $n$th power one obtains the matrix for propagation through an $n$-segment waveguide of length $d$. The $n$th power of a $2 \times 2$ matrix is ${ }^{12}$

$$
\left(\begin{array}{ll}
A & B \\
C & D
\end{array}\right)^{n}=\frac{1}{\sin \theta}\left\{\begin{array}{cc}
A \sin (n \theta)-\sin [(n-1) \theta] & B \sin (n \theta) \\
C \sin (n \theta) & D \sin (n \theta)-\sin [(n-1) \theta]
\end{array}\right\}
$$

$$
\begin{aligned}
L_{10} & =\frac{I_{0} \int_{0}^{2 \pi} \int_{w_{m}}^{\infty} \cos ^{2} \theta \exp \left(-2 r^{2} / w^{2}\right) r^{3} d r d \theta}{I_{0} \int_{0}^{2 \pi} \int_{0}^{\infty} \cos ^{2} \theta \exp \left(-2 r^{2} / w^{2}\right) r^{3} d r d \theta} \\
& =\left(1+\frac{2 w_{m}^{2}}{w^{2}}\right) \exp \left(-\frac{2 w_{m}^{2}}{w^{2}}\right) \\
& =\left[1+2 \pi N\left(\frac{\lambda d}{\pi w^{2}}\right)\right] \exp \left[-2 \pi N\left(\frac{\lambda d}{\pi w^{2}}\right)\right] .
\end{aligned}
$$

In Fig. 6 is a plot from Ref. 10 of the single pass diffraction losses of the TEM TO $_{00}$ mode for various resonator types. The Gaussian approximations from Eq. (22) are plotted as dashed lines. Semiquantitative agreement is obtained for all values of the Fresnel number $N$. Other analytic approximations have been given previously. For example, it has been shown that for large values of the Fresnel number, the losses of plane parallel and concentric resonators are given by ${ }^{11}$

$$
L_{n m}=8 \nu_{n m}^{2} \frac{\beta(M+\beta)}{\left[(M+\beta)^{2}+\beta^{2}\right]^{2}},
$$

where $\nu_{n m}$ is the $m$ th zero of the Bessel function $J_{n}(x), \beta=0.824$, and $M=(8 \pi N)^{1 / 2}$. This result is plotted as a dotted line in Fig. 6, and the approximation apparently fails for Fresnel numbers below $N \simeq$ 1. The corresponding results for the TEM 10 mode are plotted in Fig. 7. Thus besides yielding a qualitative description of the field distribution, the Gaussian approximation also provides an estimate of the resonator losses. The method is general and applies as well to resonators containing an arbitrary assortment of lenses and lenslike media. In addition these analytic solutions give a useful first estimate for numerical mode iterations.

\section{Mode Selection in Apertured Waveguides}

The matrix methods that we have described may readily be applied to find the properties of optical waveguides containing lenses, apertures, and lenslike media. Relationships are derived between waveguides containing discrete lens and aperture elements and waveguides containing complex lenslike media. The transverse mode discrimination characteristics of apertured waveguides are also considered.

In analogy with Eq. (12), the matrix governing. beam propagation through a lens of focal length $f$ followed by an aperture of Gaussian spot size $w_{m}$ and a length $d / n$ of free space is

$$
\left(\begin{array}{ll}
A & B \\
C & D
\end{array}\right)=\left(\begin{array}{cc}
1-\frac{d}{n f}-i \frac{\lambda d}{n \pi w_{m}^{2}} & \frac{d}{n} \\
-\frac{1}{f}-i \frac{\lambda}{\pi w_{m}^{2}} & 1
\end{array}\right) .
$$

where $\theta=\cos ^{-1}[(A+D) / 2]$. If the conditions $|f| \gg$ $d / n$ and $\left|n w_{m}{ }^{2} / \lambda d\right|=|N| \gg \pi^{-1}$ are both satisfied, one obtains from Eq. (26)

$$
\theta=\left(\frac{d}{n f}+i \frac{\lambda d}{n \pi w_{m}^{2}}\right)^{1 / 2} .
$$

Since $A \simeq D=1$, Eq. (27) may be written

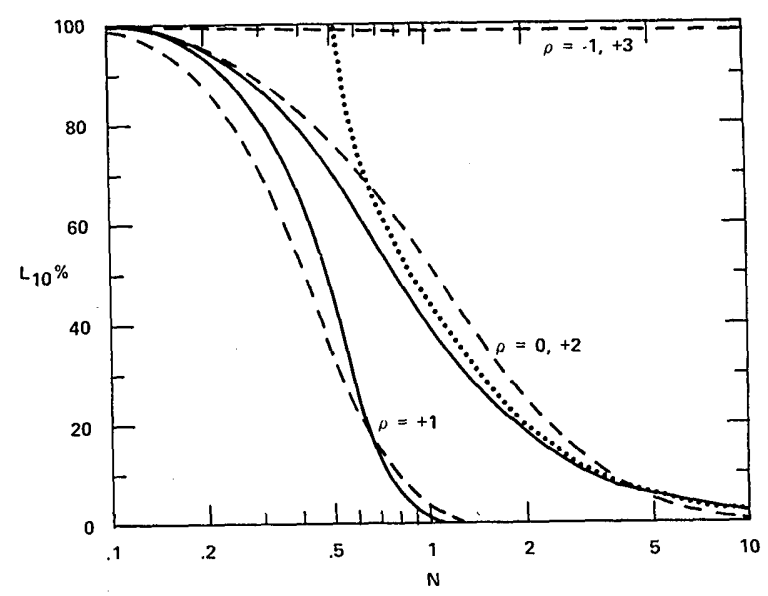

Fig. 7. Power transmission $L_{10}$ of the $\mathrm{TEM}_{10}$ mode as a function of the Fresnel number for various values of $\rho=d / R_{m}$. The exact numerical solutions are shown as solid lines, the analytic Gaussian mode approximations are shown as dashed lines, and a previous approximation is shown as a dotted line.

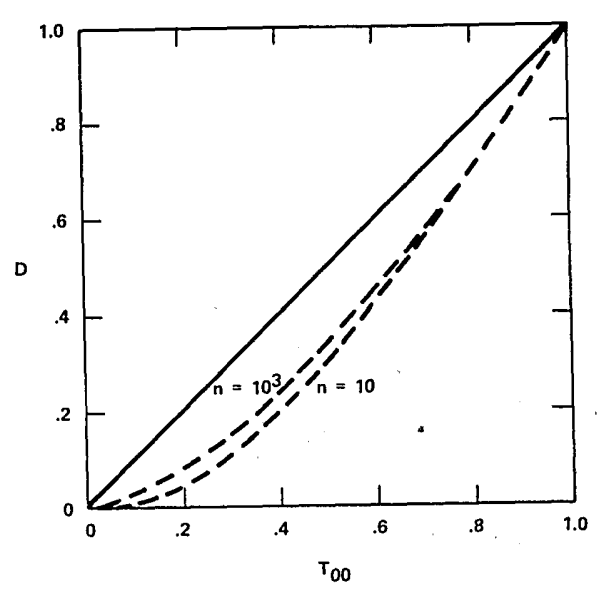

Fig. 8. Mode discrimination parameter $D=T_{10} / T_{00}$ for a waveguide with Gaussian profiled transmission apertures (solid line). The corresponding results for abrupt apertures are also shown (dashed lines) for $n=10$ and $n=1000$. 


$$
\left(\begin{array}{ll}
A & B \\
C & D
\end{array}\right)^{n} \simeq \frac{1}{\sin \theta}\left\{\begin{array}{cc}
2 \sin \left(\frac{\theta}{2}\right) \cos \left[\left(n-\frac{1}{2}\right) \theta\right] & B \sin (n \theta) \\
C \sin (n \theta) & 2 \sin \left(\frac{\theta}{2}\right) \cos \left[\left(n-\frac{1}{2}\right) \theta\right]
\end{array}\right\} .
$$

But the magnitude of $\theta$ is small compared to unity, and when $n$ is large this result reduces to

$$
\begin{aligned}
& \left(\begin{array}{ll}
A & B \\
C & D
\end{array}\right)^{n} \simeq\left[\begin{array}{ll}
\cos (n \theta) & \frac{B}{\theta} \sin (n \theta) \\
\frac{C}{\theta} \sin (n \theta) & \cos (n \theta)
\end{array}\right] \\
& =\left\{\begin{array}{rr}
\cos \left[\left(\frac{n}{f d}+\frac{i n \lambda}{\pi w_{m}^{2} d}\right)^{1 / 2} d\right] & \left(\frac{n}{f d}+\frac{i n \lambda}{\pi w_{m}^{2} d}\right)^{-1 / 2} \sin \left[\left(\frac{n}{f d}+\frac{i n \lambda}{\pi w_{m}^{2} d}\right)^{1 / 2} d\right] \\
-\left(\frac{n}{f d}+\frac{i n \lambda}{\pi w_{m}^{2} d}\right)^{1 / 2} \sin \left[\left(\frac{n}{f d}+\frac{i n \lambda}{\pi w_{m}^{2} d}\right)^{1 / 2} d\right] & \cos \left[\left(\frac{n}{f d}+\frac{i n \lambda}{\pi w_{m}^{2} d}\right)^{1 / 2} d\right]
\end{array}\right\} .
\end{aligned}
$$

Equation (30) is identical in form to the matrix for a complex lenslike medium shown in Fig. 1. Therefore, one can make the identifications

$$
\begin{gathered}
\beta_{2}=2 \pi n / \lambda d f, \\
\alpha_{2}=2 n / w_{m}^{2} d,
\end{gathered}
$$

where the complex propagation constant varies radially according to $k=k_{0}-k_{2} r^{2} / 2, \beta_{2}$ and $\alpha_{2}$ are the real and imaginary parts of $k_{2}$, and the gain per wavelength is small $\left(k_{0} \simeq \beta_{0}=2 \pi / \lambda\right)$. Thus in this limit of long focal length and large aperture diameter the lens-aperture waveguide is mathematically equivalent to a complex lenslike medium. This information simplifies the analysis of some waveguide problems, and waveguides consisting of only lenses or only apertures are special cases of these results. By analogy with the discussion of Sec. II, one can also conclude that the addition of Gaussian apertures to a waveguide leads to stabilization against perturbations of the spot size, phase front curvature, and beam displacement provided that $w_{m}^{2}>0$. The stabilizing effect of graded apertures may be useful in optical communications and for stabilizing the amplifier chains of laser function devices.

The perturbations of the parameters of a fundamental TEM $_{00}$ Gaussian beam can be expanded in terms of higher order Gaussian beam modes. Thus a stable waveguiding system should show a strong discrimination against the $\mathrm{TEM}_{10}$ mode. Using the previously described beam methods this mode selection can be verified analytically. For simplicity we assume that the waveguiding is accomplished only by $n$ equally spaced apertures, and waveguides including lenses can be treated similarly. The transmission of the fundamental $\mathrm{TEM}_{00}$ mode through a periodic configuration is given by $T_{00}=\left(1-L_{00}\right)^{n}$, where $L_{00}$ is the single pass leakage past a graded mirror derived in Sec. II (which is equivalent to the absorption in a graded aperture). We define a mode discrimination parameter $D$ by

$$
D=\frac{T_{10}}{T_{00}}=\frac{\left(1-L_{10}\right)^{n}}{\left(1-L_{00}\right)^{n}}
$$

A.small value of $D$ implies good selectivity.
For Gaussian apertures the expressions for $L_{00}$ and $L_{10}$ are given in Eqs. (17) and (19), and one obtains from Eq. (33)

$$
D=T_{00}=\left(1+w^{2} / w_{m}^{2}\right)^{-n}=\left[1+(\pi N)^{-1}\left(\pi n w^{2} / \lambda d\right)\right]^{-n} .
$$

Thus the transmission $T_{10}$ is simply equal to $T_{00}{ }^{2}$ for all values of $n$ and $N$, and this result is plotted as a straight line in Fig. 8. When $N$ is large compared to $\pi^{-1}$, the aperture spot size is found to be $\left(\pi n w^{2} / \lambda d\right)$ $\simeq(2 \pi N)^{1 / 2}$, and Eq. (34) can be written

$$
D \simeq\left[1+(2 / \pi N)^{1 / 2}\right]^{-n} \simeq \exp \left[-n(2 / \pi N)^{1 / 2}\right] .
$$

In this limit the aperture waveguide can be regarded as a complex lenslike medium with $\alpha_{2}$ given in Eq. (32), and Eq. (35) can be obtained from the theory of beam propagation in complex lenslike media.

As an example, we consider the case of a long aperture-guided 10.6- $\mu \mathrm{m} \quad \mathrm{CO}_{2}$ laser amplifier. After every meter of amplification, we assume that there is a Gaussian transmission filter having a spot size $w m=$ $1 \mathrm{~cm}$. This corresponds to a Fresnel number of $N=$ $\omega \mathrm{m}^{2} / \lambda d=9.43$. From Fig. 2 one finds that the beam spot size just before an aperture is $\left(\pi w^{2} / \lambda d\right)=8.7$. The transmission loss at an aperture from Fig. 4 is $L_{00}=23 \%$, which is small compared to the laser gain of about $5 \mathrm{~m}^{-1}{ }^{13}$ From Eq. (34) the mode discrimination in one amplifier stage is $D=\left(1+w^{2} / w m^{2}\right)^{-1}=$ 0.77 so the higher order mode is only weakly damped. The stability factor of Fig. 5 is $F=1.15$. Therefore, perturbations of the spot size or phase front curvature will be reduced after $1 \mathrm{~m}$ by the factor $F^{-2}=$ 0.76 , and perturbing beam displacements will be reduced by $F^{-1}=0.87$. In this large- $n$ limit useful approximations include $\left(\pi w^{2} / \lambda d\right) \simeq(2 \pi N)^{1 / 2}, L_{00} \simeq[1$ $\left.+(\pi N / 2)^{1 / 2}\right]^{-1}$, and $F \simeq 1+(2 \pi N)^{-1 / 2}$.

Waveguiding is also possible with abruptly profiled transmission filters. For the loss in one section of such a waveguide Eq. (25) may be used, and Eq. (33) becomes

$$
D=\left[\frac{1-\left(\nu_{10} / \nu_{00}\right)^{2} L_{00}}{1-L_{00}}\right]^{n} \text {. }
$$


The loss $L_{00}$ can be expressed in terms of $T_{00}$, and Eq. (36) reduces to

$$
D=T_{00}{ }^{-1}\left[1-\left(\nu_{10} / \nu_{00}\right)^{2}\left(1-T_{00}{ }^{1 / n}\right)\right]^{n}
$$

This formula is plotted in Fig. 8 for $n=10$ and $n=$ 1000 . For small values of $n$, Eq. (25) is not valid. When $n$ is equal to unity the numerical loss results for a plane parallel resonator can be used,,$^{10}$ and the results are similar to those shown in the figure.

It is clear from Fig. 8 that increasing the number of apertures in an aperture waveguide does not have a significant effect on the mode discrimination. Periodic bore ripples are, however, sometimes introduced in practical high gain $\mathrm{CO}_{2}$ amplifiers to quench the persistent TEM $_{10}$ mode. ${ }^{14}$ In this case the higher order mode is otherwise apparently selectively enhanced by the wall interaction and the gain profile.

\section{v. Conclusion}

A matrix method has been described for investigating the properties of laser resonators and waveguides with large diffraction losses. The method yields exactly the spot size phase front curvature, stability, and loss characteristics of the modes of laser resonators with Gaussian graded reflectivity profiles. Arbitrary assortments of lenses and lenslike media can also be included. In addition a useful analytic approximation is obtained for the properties of lasers with abrupt mirror reflectivity profiles. Unlike previous approximations, agreement is obtained for all values of the Fresnel number, and the results also apply to periodic optical waveguides.

This work was supported in part by the National Science Foundation.

\section{References}

1. A. G. Fox and T. Li, Bell Syst. Tech. J. 40, 453 (1961).

2. A. E. Siegman and H. Y. Miller, Appl. Opt. 9, 2729 (1970).

3. G. L. McAllister, W. H. Steier, and W. B. Lacina, IEEE J. Quantum Electron. QE-10, 346 (1974) and references.

4. N. G. Vakhimov, Radio Eng. Electron. Phys. 10, 1439 (1965).

5. H. Zuker, Bell Syst. Tech. J., 49, 2349 (1970).

6. H. Kogelnik and T. Li, Appl. Opt. 5, 1550 (1966).

7. Y. Suematsu, K. Iga, H. Nagashima, and T. Kitano, Electron. Commun. Japan 51-B, 67 (1968).

8. L. W. Casperson, Appl. Opt. 12, 2434 (1973).

9. L. W. Casperson, IEEE J. Quantum Electron. QE-10, 629 (1974).

10. T. Li, Bell Syst. Tech. J. 44, 917 (1965).

11. L. A. Vainshtein, Sov. Phys. JETP 17, 709 (1963).

12. M. Born and E. Wolf, Principles of Optics (Pergamon, New York, 1970), p. 67.

13. O. R. Wood, IEEE Proc. 62, 355 (1974).

14. O. M. Stafsudd, UCLA; private communication.

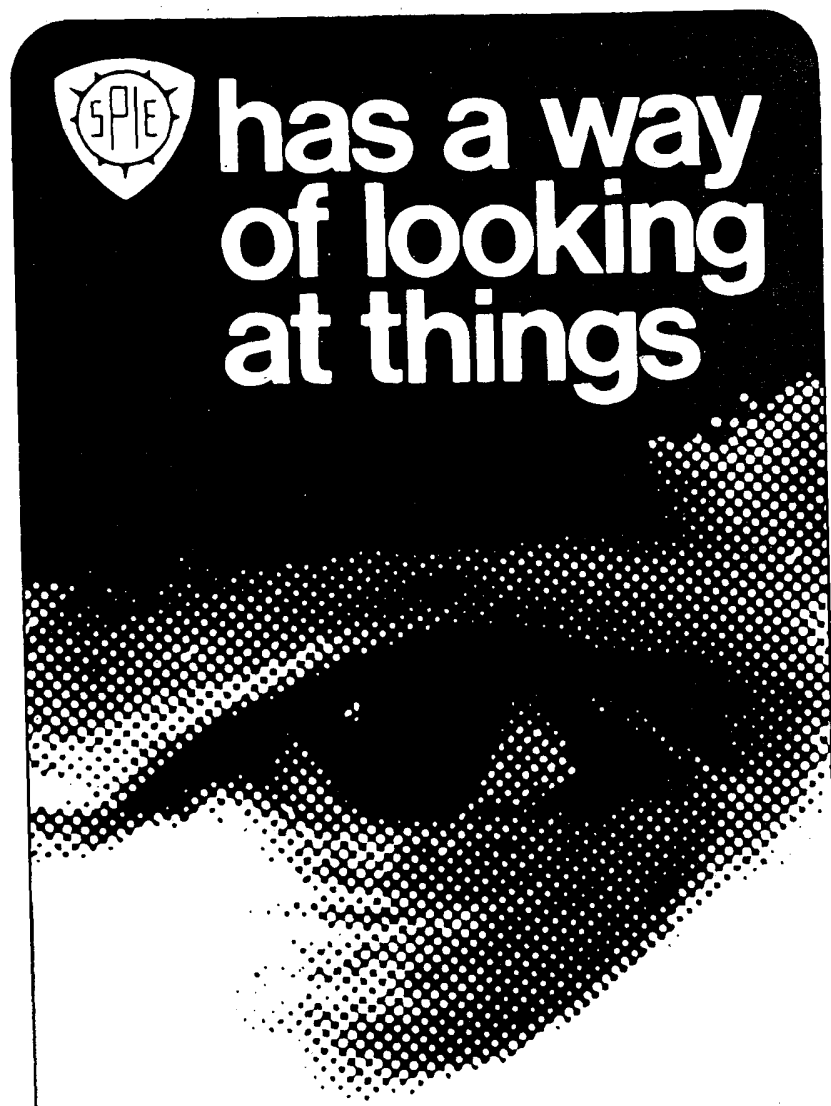

2na European

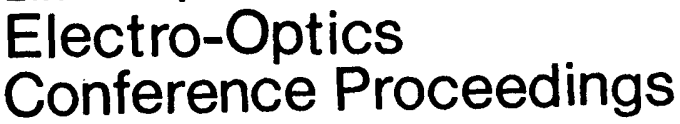

Meeting held April 2-5, 1974, Montreux, Switzerland 350 pages - Price $\$ 51.00$ (U.S. \& Canada only)

Published by Mack-Brooks Exhibitions Ltd., England, in cooperation with the Society of Photo-Optical Instrumentation Engineers, USA.

Cosponsored by The Institute of Applied Physics of the Swiss Federal Institute of Technology; European Physical Society; The Smithsonian Institution.

The aim was to provide an expertly digested review of developments in Electro-Optics Technology and at the same time provide an informal forum for the free exchange of ideas and opinions. The papers reflected those developments and changes of emphasis that have occurred within the industry since the first Geneva conference in 1972. In particular, uranium enrichment using tunable lasers was covered for the first time, as was laser-triggered nuclear fusion. Over 80 papers were presented giving topical information on the following areas: Lasers, Present \& Future; Radiation Detection \& Spectroscopy; Laser-Induced Chemical Effects; Integrated Optics, Waveguides, Fibres; Environmental Protection; Electro-Optical Crystals \& Materials; Electro-Optical Storage, Memories, \& Holography; Testing, Measuring, \& Materials Processing; Opto-Electronics Sensing \& Display Components; Low Light Opto Eystems; Electro-Optical Recording \& Processing; Progress in Laser Nuclear Fusion Technology; Optical Communications; Electro-Optics in Biology \& Medicine.

ALL ORDERS MUST BE PREPAID. Price includes shipping and handling. Within California add $6 \%$ sales tax.

Make checks payable to:

SOCIETY OF PHOTO.OPTICAL INSTRUMENTATION ENGINEERS. P.O. Box 1146, Palos Verdes Estates, California 90274 Telephone: (213) 378-1216. 